\title{
Sensitivity and Responsiveness of Ibadan Stroke-Specific Pain Scale
}

\author{
Osundiya O.C., ${ }^{1}$ Owolabi M.O., ${ }^{2}$ Hamzat T.K. ${ }^{2}$ \\ ${ }^{1}$ Physiotherapy Department, University College Hospital, Ibadan \\ ${ }^{2}$ College of Medicine, University of Ibadan \\ Correspondence \\ Osundiya Oladunni C. PhD, Physiotherapy Department, University College Hospital, Ibadan \\ (cosundiya@yahoo.com)
}

\begin{abstract}
SUMMARY
Sensitivity and responsiveness to change are important psychometric properties of outcome measures, especially in evaluating therapeutic effectiveness (Dromeric and Redeng, 2003). The consequences of an outcome measure that lacks responsiveness are the same as a diagnostic test that has poor sensitivity or specificity (Scrimshaw and Maher, 2001). The ability of an instrument to be sensitive to within patient change is very important in clinical trials especially in a disease like stroke where the impact is often life-long and multi-dimensional (Poissant et al, 2003).

The purpose of this study therefore was to investigate the responsiveness of the Ibadan Stroke-Specific Pain Scale (IbSSPS) in evaluating post-stroke pain.

Fifty-six patients with first-incidence stroke experiencing post-stroke pain were assessed for pain. Sensitivity to change was assessed by analysing changes in the IbSSPS scores before and after six weeks of physiotherapy with the Wilcoxon-sign rank test. Standardized effect size (SES) and standardized response mean (SRM) were used to assess responsiveness.

The overall IbSSPS and its four domains were sensitive to change after 6 weeks of physiotherapy. It was therefore concluded that the IbSSPS is a sensitive and responsive scale that can be used to evaluate pain in stroke survivors.
\end{abstract}

Key words: Post-stroke pain, Stroke-specific Pain Scale, sensitivity, responsiveness

\section{INTRODUCTION}

Recovery and long-term health of stroke survivors can be adversely affected by a number of medical complications, including chronic pain (Yang et al, 2009). However, because of the subjective nature of pain, clinical importance is not always easy to determine (Farrar et al, 2001).

Pain which is one of the most common and highly challenging medical problems in health care is one of the most frequently observed complications of stroke (Zorowitz et al, 2005). Though it is increasingly recognized as a consequence of stroke (Klit at al, 2011; Miller et al, 2013) and may be serious enough to jeopardize recovery by hindering rehabilitation (Jonsson et al, 2006), its assessment is fundamental in selecting an appropriate therapy and modifying therapy according to the individual patient's response (Zeferino and Aycock, 2010).

Generic scales often do not tap attributes that are relevant to stroke patients and exhibit low sensitivity; hence the need for a stroke-specific measure (Owolabi, 2010). They are not designed for specific diseases and are thus not sensitive to subtle patient-specific and disease-specific changes (Williams et al, 1999 Owolabi, 2010). Generic measures lack adequate content validity for stroke, they are not suitable for routine clinical use or clinical trials in 
stroke (Owolabi, 2010) while disease-specific measures have been found to quantify the impact of a specific pain problem on function and can be used to track changes after an intervention (Garrat et al, 2001).

Responsiveness is the ability of a measure to detect a clinically important and meaningful change while sensitivity shows a statistically significant change irrespective of the relevance or meaningfulness of such change (Liang et al, 2002). Using sensitive and responsive measures enhance clinical practice (van der Putten et al, 1999).

A newly developed stroke-specific pain scale - the Ibadan Stroke Specific Pain Scale (IbSSPS), which combines both self-report and clinician report has no reports yet on its responsiveness and sensitivity. These are prerequisites for its utility as an outcome measure (Terwee et al, 2007) hence, this study evaluated the responsiveness and sensitivity to change of the IbSSPS.

\section{A Priori Statement}

To determine the sensitivity to change of the IbSSPS, it was hypothesized that it will report statistically significant differences in IbSSPS scores after a six-week intervention. A second hypothesis was that the IbSSPS will be sensitive to change in the pain status of the survivors after 6 weeks of treatment.

\section{METHODS}

This was a prospective study of stroke survivors with not more than 12 months stroke onset. Participants were consecutively recruited patients from the outpatient physiotherapy clinic of the University College Hospital, who were able to communicate and willing to participate. Ability to communicate was assessed by three word commands, e.g. 'Make a fist', 'Touch your nose'. Patients with severe cognitive deficits and co-morbidities such as cancer were excluded from the study. The study was conducted between May 2012 and January 2013. Of the 64 stroke patients recruited into the study, 8 were lost to follow-up and were therefore excluded from the study.

\section{Data Collection}

Ethical approval was granted by the University of Ibadan/University College Hospital, Ethical Committee.

Data on age, sex, stroke laterality, stroke onset, pain onset and IbSSPS score were obtained at baseline, while the IbSSPS was re-administered after 6 weeks of physiotherapy.

\section{The Ibadan Stroke Specific Pain Scale (IbSSPS)}

The IbSSPS is a multidimensional stroke-specific pain scale which combines a self and clinician's report. It contains 36 items in 4 domains (Pain location/Severity, Psychosocial functioning, Physical functioning and Signs and Symptoms). Items in the first 3 domains are scored on a 5-point Likert scale. The items in the fourth domain which is a clinician's report is scored absent or present. It is evaluative, revealing the presence or absence of signs and symptoms of different types of post-stroke pain and hence not summed.

Patients respond to each item using the corresponding response set as indicated. Scores in each domain are summed up while the total IbSSPS score is a summation of the first 3 domains with a maximum score of 144, with higher scores indicating higher pain status.

\section{Data Analysis}

Data were screened for normality. Non-normality was addressed using appropriate transformations. Descriptive statistics of mean, standard deviation, frequencies and percentages were used to summarize the demographic and clinical data of the stroke patients.

Sensitivity was determined by examining the changes in domain and overall IbSSPS scores between the baseline and after 6 weeks of physiotherapy using the Wilcoxon signed rank test.

Responsiveness was assessed using the standardized effect size (SES) and standardized response mean (SRM) (Kazis et al, 1989). The SES was calculated as mean difference in IbSSPS scores between the baseline and 6 weeks (Kazis et al, 1989), while the standardized response mean (SRM) was calculated as the ratio of mean change to the standard deviation of that change.

SRM or SES of $<0.2$ depicts no responsiveness, $0.2-$ 0.5 mild responsiveness, 0.5 - 0.71 moderate responsiveness, while a SRM or SES greater than 0.7 represents marked responsiveness (Cohen, 1977). The level of statistical significance was set at 0.05 .

\section{RESULTS}

Fifty-six out of sixty-four stroke survivors completed the study. The majority (68\%) had left-sided hemiplegia and $32 \%$ had right-sided hemiplegia with a duration of pain onset between 2 and 16 weeks.

Across the four domains, there was significant reduction in the IbSSPS scores of the participants after physiotherapy (table 1), this showing good sensitivity. 
Table 1. Comparison of Ibadan Stroke-Specific Pain Scale scores before and after 6-weeks of Physiotherapy using the Wilcoxonsigned rank test $(\mathrm{N}=56)$

\begin{tabular}{lcccccc}
\hline & \multicolumn{2}{c}{ Pre } & \multicolumn{2}{c}{ Post } \\
& $\mathrm{X} \pm \mathrm{SD}$ & $\mathrm{X} \pm \mathrm{SD}$ & $\mathrm{Z}$ & $\mathrm{p}$ \\
\hline $\begin{array}{l}\text { Pain } \\
\text { location/severity }\end{array}$ & 6.80 & 5.21 & 4.63 & 3.69 & -5.40 & $<0.001^{*}$ \\
$\begin{array}{l}\text { Psychosocial } \\
\text { functioning }\end{array}$ & 10.16 & 6.21 & 7.87 & 4.94 & -5.22 & $<0.001^{*}$ \\
$\begin{array}{l}\text { Physical } \\
\text { functioning }\end{array}$ & 20.79 & 11.36 & 17.69 & 9.92 & -3.72 & $<0.001^{*}$ \\
$\begin{array}{l}\text { Signs \& } \\
\text { symptoms }\end{array}$ & 3.48 & 1.18 & 2.32 & 1.65 & -3.94 & $<0.001^{*}$ \\
TOTAL & 41.51 & 18.86 & 32.41 & 15.31 & -4.98 & $<0.001^{*}$ \\
\hline
\end{tabular}

Significant at $<0.01$

The standardized effect size for the domains ranged between 0.31 and 0.70 (table 2). The standardized response mean also ranged between 0.5 and 0.9 . This connotes the ability of the IbSSPS to pick clinically relevant changes in the pain status.

Table 2. Responsiveness of IbSSPS domains after 6 weeks Physiotherapy using Standardized Effect Size and Standardized Response Mean

\begin{tabular}{lll}
\hline Domain & SES & SRM \\
\hline Pain location/severity & 0.59 & 0.90 \\
Psychosocial functioning & 0.47 & 0.8 \\
Physical functioning & 0.31 & 0.5 \\
Signs and Symptoms & 0.70 & 0.6 \\
SES - Standardized Effect Size & \\
SRM - Standardized Response Mean &
\end{tabular}

\section{DISCUSSION}

Sensitivity and responsiveness, though components of validity, have been considered separate attributes of outcome measures because of their pivotal role in clinical trials (Terwee et al, 2007). Generic pain measures are commonly used even in stroke trials despite the disadvantage that the majority of these measures are unlikely to address issues that are of great importance to the stroke survivors or capture the multidimensional nature of post-stroke pain. One of such measures is the Visual Analogue Scale (VAS), which is preferred to McGill when measuring pain in clinical trials and clinical practice because it is more responsive in assessing pain over a period of 24 hours (Scrimshaw and Maher, 2001).

This study examined the sensitivity and responsiveness to change of a stroke-specific pain scale, the IbSSPS. All the domains of the IbSSPS had at least mild responsiveness while the pain location/severity and the psychosocial functioning exhibited marked responsiveness with standardized effect size and standardized response mean (SRM) above 0.70 . This is indicative of the extent to which the scores on the scale reflect changes in the patient's condition which is expected to be in line with the direction of pain status. These changes were related to the subjective improvement or alteration of pain after 6 weeks of physiotherapy intervention.

The mild responsiveness observed in the physical functioning domain is an indication that despite the negative impact of post-stroke pain on motor functioning, the relationship between functional disability and pain levels is not always strong. Changes in pain level are not always highly correlated with changes in functional disability and vice versa (Palermo et al, 2004). This is because pain is not an independent predictor of function especially in a disabling and multifaceted disease like stroke.

The signs and symptoms domain being discriminatory and not necessarily needing to be responsive to change (Terwee et al, 2007) still demonstrated moderate responsiveness.

The IbSSPS demonstrated a good attribute of a diseasespecific measure as it was able to detect small improvements and deteriorations. This trend is similar to the one reported by Williams et al (1999) in the development and validation of a stroke-specific quality of life scale. This makes it a useful tool that can complete favourably as a stroke-specific instrument in assessing the impact of therapeutic and rehabilitative interventions in stroke patients as it is able to assess meaningful changes.

Although preferential or selective therapeutic effect was not demonstrated, the observed changes after intervention were evident for both total and sub-scores. This is contrary to the findings of Bouhassira et al (2004) in the validation of the Neuropathic Pain Scale. They found the Neuropathic Pain Scale to be responsive to changes but the changes were only evident for the total score not for the sub-scores.

The inclusion of participants with mild to moderate cognitive impairment is an area of strength for the IbSSPS because using a list of words to describe pain has been found to be better for patients with cognitive impairment (Chibnall and Tait, 2001).

One limitation of this scale, which has been found with other pain rating scales is the likely loss of data with stroke survivors with severe cognitive deficit. 


\section{CONCLUSION}

Our results suggest that the IbSSPS is a sensitive and responsive tool to assess post-stroke pain. Results show a significant change in IbSSPS score both for the total and subscale scores and further support the use of such a scale for detecting and treating pain in stroke survivors. This also confirms the clinical utility of the scale.

Overall, the IbSSPS is a novel clinical tool for assessing pain in stroke survivors, and hence would lead to significant improvement in the care of stroke survivors in the future.

\section{References}

Bouhassira D., Attal N., Fermanian J., Alchaar H., Gautron M., Masquelier E., Rostaing S., Lanteri-Minet M., Collin E., Grisart J., Boureau F. 2004. Development and validation of the Neuropathic Pain Symptom Inventory. Pain 108(3): 248257.

Chibnall J.T., Tait R.C. 2001. Pain assessment in cognitively impaired and unimpaired older adults: a comparison of four scales. Pain 92(1): 173-186.

Cohen, J. 1977. Statistical Power Analysis for the Behavioural Sciences. New York, NY: Academic Press.

Dromerick A., Redeng M. 2003. Medical and neurologic complications of stroke during inpatient rehabilitation. Stroke 25(2): 358-361.

Farrar J.T., Young Jr, J.P., LaMoreaux L. Werth, J.L., Poole, R.M. 2001. Clinical importance of changes in chronic pain intensity measured on an 11-point numerical pain rating scale. Pain 94(2): 149-158.

Fayers PM, Machin D. 2000. Quality of Life Assessment, Analysis and Interpretation. Chichester: John Wiley.

Garratt A.M., Klaber M.J., Farrin A.J. 2001. Responsiveness of generic and specific measures of health outcome in low back pain. Spine 26(1): 71-77.

Jonsson A.C., Lindgren I., Hallstrom B., Norrving B., Lindgren A. 2006. Prevalence and intensity of pain after stroke: A population-based study focusing on patients persepectives. Journal of Neurology, Neurosurgery and Psychiatry 77: 590595.

Kazis L.E., Andersennon J.J., Meenan R.F. 1989. Effect sizes for interpreting changes in health status. Med Care 27: SI78-89.

Klit H., Finnerup N.B., Overrad K., Anderson G., Jensen T.S. 2011. Pain following stroke: A population-based follow-up study. PLoS One 6(11): e27607

Liang M.H., Lew R.A., Stucki G., Fortin P.R., Daltroy L. 2002. Measuring clinically important changes with patient-oriented questionnaires. Medical Care 40(Suppl): 84-90.

Miller K.K., Combs S.A., Van Rymbroeck M., Altenburger P.A., Kean J., Dierks T.A., Schmid A.A. 2013. Fatigue and pain: Relationships with physical performance and patient beliefs after stroke. Topics in Stroke Rehabilitation 20(4): 347-355.

Owolabi M.O. 2010. Which is more valid for stroke patients: Generic or stroke-specific quality of life measures? Neuroepidemiology 34(1): 8-12.

Palermo T.M., Valenzuela D., Stork P.P. 2004. A randomized trial of electronic versus paper pain diaries in children: Impact on compliance, accuracy, and acceptability. Pain 107: 213-9.

Poissant L., Mayo N.E., Wood-Dauphinee S., Clarke A.E. 2003. The development and preliminary validation of a referencebased stroke index (PBSI). Health and Quality of Life Outcomes 1(43): 1-15.

Price C.I.M., Curless R.H., Rodgers H. 1999. Can stroke patients use Visual Analogue Scale? Stroke 30: 1357-1361.

Scrimshaw S.V., Maher C. 2001. Responsiveness of visual analogue and McGill pain scale measures. Journal of Manipulative and Physiological Therapeutics 24(8): 501-504.

Terwee C.B.,Bota D.M., de Boera, M.R., Van der Windta, D. A.W.M., Kuola, D.L., Dekkera, J.,Boutera, L.M., de Veta, H.C.W. 2007. Ouality Criteria proposed for measurement properties of Health Status Questionnaire. Journal of Clinical Epidemiology 60: 34e42.

van der Putten J.J.M.F., Hobart J.C., Freeman J.A., Thompson A.J. 1999. Measuring change in disability after inpatient rehabilitation: comparison of the responsiveness of the Bathel Index and the Functional Independence Measure. Journal of Neurology, Neurosurgery and Psychiatry 66: 480-484.

Williams L.S., Weinberger W., Harris L.E.,Clark D.O., Biller J. 1999. Development of a stroke-specific quality of life scale. Stroke 30(7): 1362-1369.

Williamson A., Hoggart B. 2005. Pain: A review of three commonly used pain rating scales. Journal of Clinical Nursing 14: 798-804.

Yang S., Grabois M., Bruel B. 2009. A clinical history and physical examination with judicious use of appropriate diagnostic modalities are mandatory in identifying what is the likely pain in stroke survivors. Practical Pain Management 9: 5.

Zeferino S.I., Aycock D.M. 2010. Post stroke shoulder pain: Inevitable or preventable? Rehabilitation Nursing 35: 4.

Zorowitz R.D., Smout R.J., Gassaway J.A., Horn, S.D. 2005. Usage of pain medications during stroke rehabilitation: The Post-Stroke Rehabilitation Outcomes Project. Topics in Stroke Rehabilitation 12(4): 37-49. 